\section{ORIGINAL RESEARCH}

\author{
W.-C. Lin \\ K.-H. Chou \\ C.-L. Chen \\ C.-H. Chen \\ H.-L. Chen
}

\section{J.A. Feekes}

N.-W. Hsu

S.-H. Li

Y.-F. Cheng

C.-P. Lin

\title{
Significant Volume Reduction and Shape Abnormalities of the Basal Ganglia in Cases of Chronic Liver Cirrhosis
}

BACKGROUND AND PURPOSE: Chronic liver disease frequently includes cognitive and movement disorders, suggesting an alteration of the striatum. With the exception of hyperintensities evident on T1-weighted images indicative of $\mathrm{Mn}$ deposition, radiographic findings of the BG are nonspecific Volumetric and morphometric analysis of DGM is limited. Whether DGM undergoes degeneration and whether this change is associated with pallidal hyperintensity and cognitive performance are currently unknown in patients with cirrhosis.

MATERIALS AND METHODS: The DGM volumes of 28 patients with chronic cirrhosis and 28 control patients were compared. Using 3D high-resolution MR images, the volume and shape of each structure were automatically analyzed by the FSL. Correlations between the DGM volume and other clinical variables, including the pallidal signal intensity, were assessed by multiple regression analysis.

RESULTS: Patients with Child B and Child $\mathrm{C}$ liver disease had significantly smaller bilateral putaminal volumes than control patients, and patients with Child $\mathrm{C}$ also demonstrated smaller left caudate nucleus and left amygdala volumes than control patients. Pallidal hyperintensity correlated with smaller striatum volume, which was linearly related to worse cognitive performance. The nonuniform distributed shape abnormalities in the striatum further support the ascending spiral interconnecting theory of the striatum.

CONCLUSIONS: These findings strongly suggest lower DGM volume develops according to the severity of the liver cirrhosis. The Mn deposition might contribute the striatum deficit. These findings support the value of additional psychomotor research associated with liver cirrhosis.

\begin{abstract}
ABBREVIATIONS: $B G=$ basal ganglia; $C A S I=$ Cognitive Ability Screening Instrument; $D G M=$ deep gray matter; FDR = false discovery rate; FIRST = Integrated Registration and Segmentation Tool of Functional MR Imaging of the Brain; GM - gray matter; GP = globus pallidus; INR = international normalized ratio; MANCOVA = multivariate analysis of covariance; $\mathrm{Mn}=$ manganese; $\mathrm{MNI}=$ Montreal Neurologic Institute; TIV = total intracranial volume
\end{abstract}

$\mathbf{P}$ atients with chronic liver disease frequently experience cognitive and movement disorders, ${ }^{1-3}$ which suggest that the striatum has been altered. ${ }^{4}$ However, most patients with liver cirrhosis show no visible abnormalities on conventional

Received January 22, 2011; accepted after revision May 9.

From the Departments of Diagnostic Radiology (W.-C.L., H.-L.C., N.-W.H., Y.-F.C.) and Surgery (C.-L.C.), Chang Gung Memorial Hospital-Kaohsiung Medical Center, Kaohsiung Chang Gung Memorial Hospital and Chang Gung University College of Medicine; Kaohsiung, Taiwan; Department of Biomedical Imaging and Radiological Sciences (W.-C.L., C.-H.C., H.-L.C., C.-P.L.), Institute of Bioengineering (K.-H.C.), and Institute of Neuroscience (C.-P.L.), National Yang-Ming University, Taipei, Taiwan; Department of Pharmacology and Physiology (J.A.F.), University of Rochester School of Medicine, Rochester, New York; and Department of Internal Medicine (S.-H.L.), Kaohsiung Chang Gung Memorial Hospital and Chang Gong University College of Medicine, Kaohsiung, Taiwan.

This work was supported by grants from the National Science Council (NSC 97-2314-B182A-104-MY3 to W.-C. Lin) and Chang Gung Memorial Hospital Research Project (CMRPG870482 to W.-C. Lin)

Ching-Po Lin and Yu-Fan Cheng contributed equally to this work.

Please address correspondence to Ching-Po Lin, PhD, Department of Biomedical Imaging and Radiological Sciences, Institute of Neuroscience, National Yang-Ming University, 155 Li-Nong St, Sec. 2, Peitou, Taipei, Taiwan; e-mail: cplin@ym.edu.tw; or Yu-Fan Cheng, MD, Department of Diagnostic Radiology, Chang Gung Memorial-Kaohsiung Medical Center, Chang Gung University College of Medicine; 123 Ta-Pei Rd, Niao-Sung Hsiang, Kaohsiung 83305, Taiwan; e-mail: cheng.yufan@msa.hinet.net

Indicates open access to non-subscribers at www.ajnr.org

Indicates article with supplemental on-line table.

http://dx.doi.org/10.3174/ajnr.A2769
MR imaging, except for hyperintensities on T1-weighted images in the $\mathrm{GP}^{5}$ and faint-to-substantial bilateral symmetric increased signal intensity along the hemispheric WM in or around the corticospinal tract on T2-weighted images. ${ }^{6}$ Pallidal hyperintensity, suggesting pathologic accumulation of Mn, has been observed. ${ }^{5,7}$ Significant elevations of Mn have also been found in other deep brain regions. ${ }^{8}$ The effect of $\mathrm{Mn}$ deposition on the GP and the DGM other than the GP remains unknown. Identifying changes in these throughout the course of liver disease may help clarify all of these psychomotor-related neurologic symptoms.

Advanced MR imaging has been used to explore the ultrastructure components of the BG in chronic liver disease. By using diffusion-weighted imaging, cerebral edema was found in the BG and even in patients with cirrhosis without encephalopathy. ${ }^{9,10}$ Persistant MR spectroscopy abnormalities after an acute encephalopathy or after liver transplantation suggest that the reversibility of the syndrome is incomplete..$^{10}$ Both cerebral edema and persistent abnormal metabolism content can result in permanent cell damage in the BG. ${ }^{11}$ However, structural imaging studies for anatomic alteration in the DGM is limited.

Volumetric techniques have been used to examine the DGM in brain development, ${ }^{12}$ aging, ${ }^{13}$ and dementia. ${ }^{14}$ In addition to volumetric assessments, structural shape analysis has also become important because of its potential to precisely 


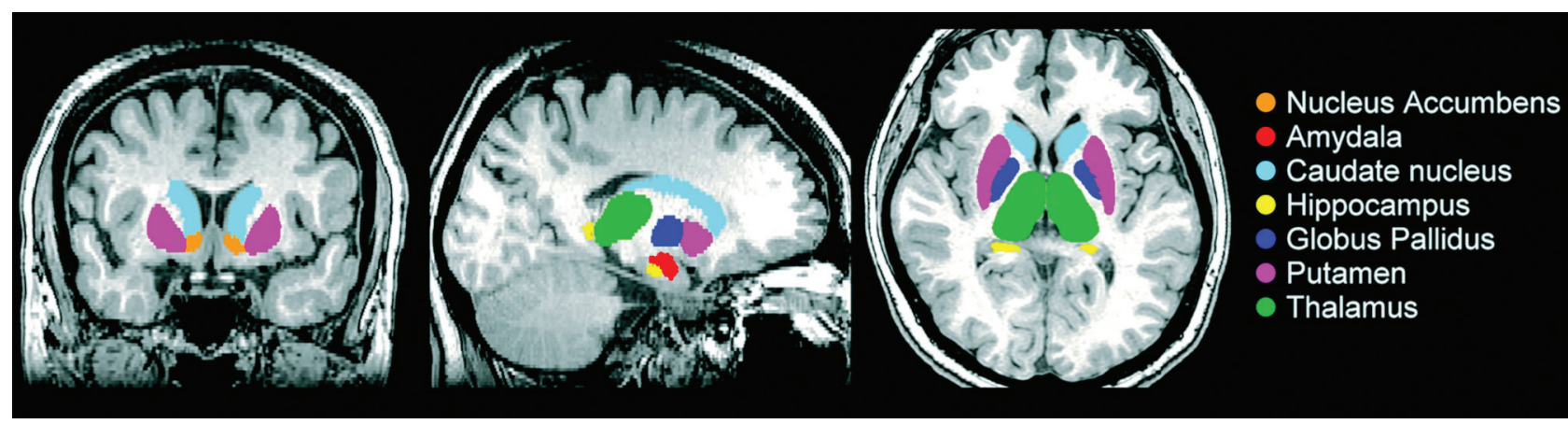

Fig 1. FIRST segmentation of the subcortical nuclei in a patient with liver cirrhosis. Volume estimation for 7 bilateral subcortical nuclei, including the nucleus accumbens, amygdala, caudate, hippocampus, pallidum, putamen, and thalamus.

locate morphologic changes between healthy and pathologic structures ${ }^{12,15,16}$ without the presence of significant volumetric differences. Both methods may clarify the disease pathogenesis and the contribution of brain structure to psychomotor decline.

We hypothesized the following: 1) Higher Child-Pugh scores are associated with smaller DGM volume; 2) correlations exist between lower DGM volume, deposition of Mn (identified from T1-weighted imaging of the pallidal index), lower laboratory profiles of liver function, and cognitive performance; and 3) local shape abnormalities might be nonuniformly distributed in cognitive and motor-related subregions.

\section{Materials and Methods}

\section{Participants}

From October 2008 to December 2009, patients with liver cirrhosis in the department of surgery for liver transplantation evaluation were prospectively enrolled. All patients presenting during this period were considered eligible. Three patients refused to participate, and 2 patients did not complete the study for technical reasons. Eight patients without qualified MR imaging studies were also excluded. Because alcohol addiction might alter the brain structure even without liver cirrhosis, ${ }^{17} 2$ patients with alcoholism-related cirrhosis were also excluded. One patient with grade IV overt encephalopathy by the West Haven criteria ${ }^{18}$ and 1 patient requiring sedation for MR imaging were also excluded. Twenty-eight patients ( 21 men, 7 women; mean age, 54 years; range, 26-70 years) with viral liver cirrhosis were studied. Cirrhosis was diagnosed on the basis of suggestive clinical and imaging features. ${ }^{19}$ The functional status of the patients was assessed via the Child-Pugh score. ${ }^{20}$ Sixteen patients were classified as Child B and 12 as Child C class. Laboratory screening was performed on the same day as the MR imaging.

Twenty-eight sex- and age-matched healthy volunteers ( 21 men, 7 women; median age, 54 years; range, $26-70$ years) were recruited. All subjects within this study with a history of drug abuse, psychiatric or neurologic illness, and head injury were excluded. The project was approved by our institutional review board, and all subjects gave written informed consent before participating.

\section{Neuropsychological Tests}

$\mathrm{CASI}^{21}$ score was obtained in all subjects. The CASI, developed as a brief screening cognitive assessment test, has 9 domains of cognitive function with scores ranging from 0 (worst) to 100 (best). CASI is the most popular instrument to rapidly detect an adult's cognitive decline.

\section{MR Imaging Data Acquisition}

The imaging data from each participant were obtained by using a $3 \mathrm{~T}$ Signa MR imaging scanner (GE Healthcare, Milwaukee, Wisconsin). A 3D fluid-attenuated inversion recovery fast-spoiled gradient recalled-echo pulse sequence was used to obtain 110 contiguous axial 1.3 - $\mathrm{mm}$ sections $(\mathrm{TR}=9.5 \mathrm{~ms}$; $\mathrm{TE}=3.9 \mathrm{~ms}$; $\mathrm{TI}=450 \mathrm{~ms}$; flip angle $=20^{\circ} ; \mathrm{FOV}=256 \times 256 \mathrm{~mm}$; matrix size $=512 \times 512$ ).

\section{Image Processing}

DGM Volume Estimation. Volume estimation for 7 bilateral DGM structures, including the nucleus accumbens, amygdala, caudate, hippocampus, pallidum, putamen, and thalamus, was performed by using FIRST, Version 1.2 (FMRIB's Integrated Registration and Segmentation Tool http://www.fmrib.ox.ac.uk/fsl/first/ index.html) which is part of FSL Version 4.1.5 (FMRIB Software Library). ${ }^{22}$ FIRST, a probabilistic adaptation of the active appearance model, ${ }^{23}$ is a completely automatic segmentation and registration tool for DGM. The shape and appearance model of each DGM structure used in FIRST is constructed from manually segmented images of 336 subjects provided by the Center for Morphometric Analysis, Massachusetts General Hospital, Boston. The manual labels are parameterized as surface meshes by a deformable surface and modeled as a point distribution function. Using a set of fixed triangles, each DGM surface mesh was reconstructed. The apex of the adjoining triangles is called a vertex. To fit the shape and appearance model to each participant's T1 image and to perform DGM tissue segmentation, we used a robust DGM structure alignment consisting of a 2-stage linear registration. The first stage is a standard whole-brain 12- $d$ faffine registration to the MNI152 template. The second stage of DGM registration applies $12-d f$ registration by using the MNI152 subcortical mask to include voxels within the DGM regions. After registration, boundary correction and mode selection with default settings were used to determine each of the final DGM segments. These segments were projected back into individual native spaces by inverse transformation matrices from the initial registration procedures, and the absolute DGM volumes were calculated (Fig 1). An expert radiologist verified the accuracy of each participant's native space DGM segments, and no errors were identified.

TIV. TIV was estimated by Gaser's VBM5 toolbox (http://dbm. neuro.uni-jena.de/vbm) with statistical parametric mapping (SPM5, Wellcome Department of Imaging Neuroscience, London, UK; http://www.fil.ion.ucl.ac.uk/spm) and implemented in Matlab 7.3 (MathWorks, Natick, Massachusetts). Whole-brain original T1weighted images were normalized, bias-field-corrected, and tissueclassified into GM, WM, and CSF within the same unified segmenta- 


\begin{tabular}{|c|c|c|c|c|c|}
\hline Group & Control & Child B & Child C & $F$ or $\chi^{2}$ & $P$ Value \\
\hline No. of subjects & 28 & 16 & 12 & & \\
\hline Age (yr) & $54.21 \pm 10.85$ & $52.75 \pm 7.80$ & $56.17 \pm 11.47$ & 0.383 & .684 \\
\hline Sex & $7 \mathrm{~F} / 21 \mathrm{M}$ & $3 \mathrm{~F} / 13 \mathrm{M}$ & $4 \mathrm{~F} / 8 \mathrm{M}$ & 0.778 & .678 \\
\hline Education (yr) & $11.75 \pm 4.34$ & $10.75 \pm 3.53$ & $8.83 \pm 3.19$ & 2.350 & .105 \\
\hline Type of hepatitis & & $12(\mathrm{~B}) / 4(\mathrm{C})$ & $9(\mathrm{~B}) / 3(\mathrm{C})$ & 0.761 & .672 \\
\hline TIV $\left(\mathrm{cm}^{3}\right)$ & $1521.86 \pm 129.58$ & $1528.18 \pm 164.67$ & $1461.36 \pm 182.17$ & 0.272 & .763 \\
\hline $\mathrm{GM}\left(\mathrm{cm}^{3}\right)$ & $658.27 \pm 60.28$ & $652.71 \pm 55.62$ & $626.14 \pm 85.14$ & 0.310 & .735 \\
\hline WM $\left(\mathrm{cm}^{3}\right)$ & $467.61 \pm 52.68$ & $471.96 \pm 73.61$ & $426.86 \pm 57.58$ & 1.222 & .303 \\
\hline Creatinine (mg/dL) & - & $0.82 \pm 0.30$ & $0.70 \pm 0.27$ & 1.148 & .294 \\
\hline Aspartate aminotransferase (IU/L) & - & $88.70 \pm 32.00$ & $105.57 \pm 31.46$ & 0.491 & .490 \\
\hline Bilirubin $(\mathrm{mg} / \mathrm{dL})$ & - & $1.34 \pm 0.87$ & $4.63 \pm 2.01$ & 28.133 & $<.001^{\mathrm{a}}$ \\
\hline Albumin (mg/dL) & - & $3.56 \pm 0.57$ & $2.75 \pm 0.38$ & 13.045 & $.001^{\mathrm{a}}$ \\
\hline INR & - & $1.14 \pm 0.13$ & $1.37 \pm 0.21$ & 12.408 & $.002^{\mathrm{a}}$ \\
\hline Venous ammonia (mg/dL) & - & $103.25 \pm 37.51$ & $147.08 \pm 102.02$ & 2.526 & .124 \\
\hline Pallidal index & $0.90 \pm 0.05$ & $1.03 \pm 0.96$ & $1.04 \pm 0.82$ & 24.406 & $<.001^{\mathrm{a}}$ \\
\hline CASI score $(0-100)$ & $97.39 \pm 4.99$ & $82.27 \pm 25.09$ & $71.00 \pm 23.86$ & 7.268 & $.002^{\mathrm{a}}$ \\
\hline
\end{tabular}

Note:-indicates not available.

${ }^{a}$ Indicates threshold for statistical significance was set at $P<0.05$

tion-generative model. This segmentation procedure was further refined by applying an iterative hidden Markov random field model to minimize the influence of the noise level during the segmentation procedure and to improve the quality of tissue segmentation. Using the tissue-classified partitions, we estimated the overall tissue volume in cubic centimeters by counting the voxels representing GM, WM, and CSF in their native space. The TIV was determined as the sum of the GM, WM, and CSF volume.

\section{Vertex-Based Shape Analysis}

In addition to volume reduction, evidence suggests physical tension from neurodegenerative mechanisms leads to shape variations. ${ }^{16,24}$ To clarify regional BG morphologic changes, we conducted vertexbased shape analyses to identify local shape differences in the putamen, caudate, and GP. These analyses were carried out via FIRST. ${ }^{23}$ Meshes with fixed numbers of vertices were reconstructed in the native spaces to encode the spatial information from all participants. However, the shapes and sizes of participants' DGM were not identical; thus, a 7- $d f$ transformation (rigid body with global scaling) was used to remove the effects from the meshes before group comparisons.

\section{Calculating the Pallidal Index}

For each participant's axial T1-weighted image, a section clearly displaying the GP location was selected, and the frontal WM area within that section was considered a reference structure. The regions of interest were identified within a $3 \times 3$ pixel box inside the central GP and frontal WM area by a radiologist without prior knowledge of the exposure status. The mean pallidal index was calculated as the average ratio of GP signal intensity to the frontal WM bilaterally. ${ }^{25}$

\section{Statistical Analysis}

Demographic Data and Clinical Variables. Comparisons for age, education level, and laboratory data among groups were conducted by using 1-way independent ANOVA tests. Categoric variables between groups, including sex and type of hepatitis, were analyzed by $\chi^{2}$ tests.

Global and Regional Tissue-Volume Comparisons. Statistical differences in the size of DGM among the groups were estimated by a MANCOVA model with each participant's age, sex, education level, and TIV as covariates. The MANCOVA model was defined by 1 be- tween-subject factor and 14 dependent variables (each bilateral of the DGM). For comparing GM, WM, and TIV, age, sex, and education level were treated as covariates. Post hoc univariate tests with the Bonferroni correction were performed to follow the significant main effects yielded by the MANCOVA test $(P<.05)$.

\section{Vertex-Based Statistical Analysis}

Group-level shape difference maps were computed between any 2 groups on a per-vertex basis. A multivariate general linear model was used to obtain F-statistics based on Pillai Trace. Each participant's age, sex, and education level were entered into the model as covariates with FDR correction $(P<.05)$.

Correlation between DGM Volume and Clinical Variables. Stepwise multiple regression analysis was used to reveal the association between clinical and demographic variables with subcortical nuclei volumes. Age, sex, education level, TIV, albumin, bilirubin, INR, creatinine, aspartate aminotransferase, venous ammonia, and pallidal indices were entered as predictors.

Correlation between DGM Volume and CASI Score. The partial correlation test was chosen for examining the relationship between DGM volume and the CASI scores, regressing out age and $\operatorname{sex}(P<.05)$. All statistical analyses were performed with the Statistical Package for the Social Sciences, Version 14.1 software (SPSS, Chicago, Illinois).

\section{Results}

\section{Clinical Characteristics and Laboratory Profiles}

The baseline characteristics of all subjects are listed in Table 1. Patients with Child C had significantly poorer laboratory test results than those with Child $\mathrm{B}$, with respect to the albumin, bilirubin, and INR value. The liver cirrhosis group (Child B and $C$ ) differed significantly from healthy subjects in pallidal intensity $(P<.001)$; however, Child $\mathrm{B}$ and Child $\mathrm{C}$ groups did not differ significantly from each other $(P=.593)$.

\section{Decreased Volume of the Putamen and Caudate Nucleus in Subjects with Cirrhosis}

The mean volume for DGM in cubic centimeters is summarized in Table 2. The MANCOVA revealed a significant difference among the 3 groups in the absolute volume of DGM $(F=1.988$, 


\begin{tabular}{|c|c|c|c|c|c|c|c|c|c|c|c|c|}
\hline \multirow[b]{3}{*}{ Anatomy } & \multicolumn{2}{|c|}{$A$} & \multicolumn{2}{|c|}{$B$} & \multicolumn{2}{|c|}{$\mathrm{C}$} & & & & & & \\
\hline & \multicolumn{2}{|c|}{ Control } & \multicolumn{2}{|c|}{ Child B } & \multicolumn{2}{|c|}{ Child C } & \multicolumn{2}{|c|}{ MANCOVA } & \multirow[b]{2}{*}{ Post Hoc Comparison } & \multicolumn{3}{|c|}{ Cohen D } \\
\hline & \multicolumn{6}{|c|}{$\overline{\text { Mean Volume (SD) }}\left(\mathrm{cm}^{3}\right)$} & $F$ & $P$ Value & & $A$ vs $B$ & B vs C & $A$ vs $C$ \\
\hline L accumbens & 0.39 & $(0.11)$ & 0.35 & $(0.09)$ & 0.30 & $(0.07)$ & 2.64 & .08 & & 0.42 & 0.69 & 1.01 \\
\hline L amygdala & 1.12 & $(0.19)$ & 1.02 & $(0.20)$ & 0.94 & $(0.23)$ & 3.12 & $.05^{\mathrm{b}}$ & $A>C ; A, B(N S) ; B, C(N S)$ & 0.51 & 0.39 & 0.87 \\
\hline L caudate & 3.09 & (0.35) & 2.92 & (0.48) & 2.70 & (0.41) & 3.53 & $.04^{\mathrm{b}}$ & $A>C ; A, B(N S) ; B, C(N S)$ & 0.39 & 0.49 & 1.01 \\
\hline L hippocampus & 3.61 & $(0.44)$ & 3.66 & $(0.41)$ & 3.51 & (0.72) & 0.11 & .90 & & -0.13 & 0.27 & 0.17 \\
\hline L pallidus & 1.78 & $(0.28)$ & 1.60 & $(0.48)$ & 1.57 & (0.56) & 1.21 & .31 & & 0.47 & 0.04 & 0.47 \\
\hline L putamen & 4.77 & $(0.54)$ & 4.33 & $(0.87)$ & 3.81 & (0.75) & 7.17 & $<.01^{\mathrm{b}}$ & $A>B ; A>C ; B, C(N S)$ & 0.60 & 0.64 & 1.47 \\
\hline$L$ thalamus & 7.24 & $(0.62)$ & 7.25 & (0.56) & 6.84 & (0.63) & 0.78 & .46 & & -0.02 & 0.69 & 0.64 \\
\hline $\mathrm{R}$ accumbens & 0.29 & $(0.09)$ & 0.26 & $(0.12)$ & 0.22 & $(0.09)$ & 1.03 & .36 & & 0.30 & 0.32 & 0.71 \\
\hline R amygdala & 1.08 & $(0.22)$ & 0.95 & (0.25) & 0.93 & $(0.20)$ & 2.59 & .09 & & 0.53 & 0.10 & 0.70 \\
\hline R caudate & 3.22 & $(0.40)$ & 3.20 & (0.52) & 2.97 & (0.42) & 0.76 & .47 & & 0.04 & 0.49 & 0.61 \\
\hline R hippocampus & 3.77 & $(0.53)$ & 3.85 & $(0.58)$ & 3.75 & $(0.38)$ & 0.06 & .94 & & -0.14 & 0.20 & 0.04 \\
\hline $\mathrm{R}$ pallidus & 1.78 & $(0.30)$ & 1.63 & (0.44) & 1.56 & $(0.46)$ & 1.13 & .33 & & 0.38 & 0.17 & 0.57 \\
\hline R putamen & 4.58 & $(0.51)$ & 4.24 & $(0.81)$ & 3.96 & (0.73) & 3.71 & $.03^{\mathrm{b}}$ & $A>B ; A>C ; B, C(N S)$ & 0.51 & 0.37 & 0.99 \\
\hline $\mathrm{R}$ thalamus & 6.99 & $(0.61)$ & 7.07 & (0.54) & 6.74 & $(0.70)$ & 0.12 & .89 & & -0.14 & 0.53 & 0.38 \\
\hline
\end{tabular}

Note:- $L$ indicates left; $R$, right; NS, no significance

${ }^{a}$ Adjusted for age, sex, education years, and TIV.

${ }^{b}$ Indicates threshold for statistical significance was set at $P<0.05$

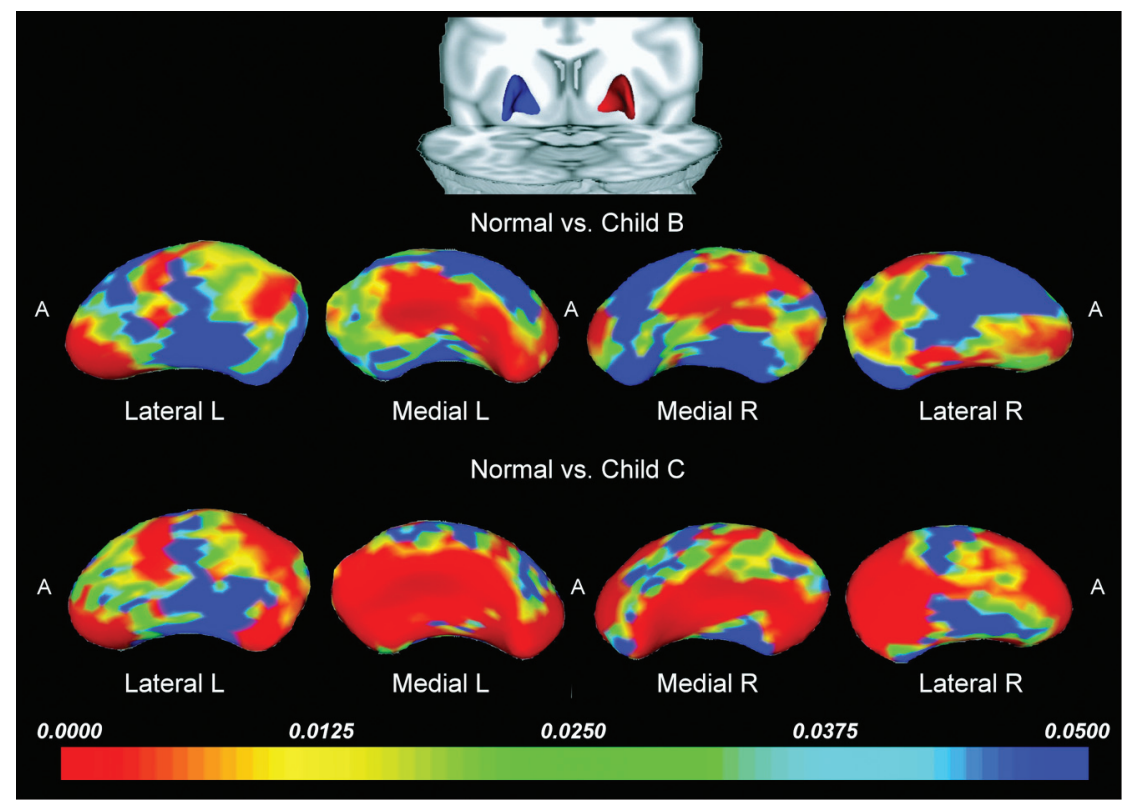

Fig 2. Statistical 3D maps revealing putaminal volume reduction in Child $B$ and $C$ groups versus controls, with $P$ values color-coded at each surface point (FDR, $P<.05$ corrected for multiple comparisons). Red areas indicate significant volume reduction $(P<.005)$; blue areas indicate little or no volume reduction $(P>.05)$. $R$ indicates right; $L$, left; $A$, anterior.

$P=.048)$. Follow-up 1-way ANCOVAs showed significant volume differences in the left putamen, right putamen, and left caudate nucleus and a trend toward a level of significance in the left amygdala among the 3 groups. Post hoc tests revealed that patients with Child $\mathrm{B}$ and Child $\mathrm{C}$ had significantly smaller volumes bilaterally in the putamen than the controls. Patients with Child C had further decrease in the left caudate nucleus and amygdala. Patients with Child C also had greater volume loss in all DGM compared with those with Child B; however, the difference was not statistically significant.

\section{Shape Analysis}

On the basis of our volumetric results, previous imaging ${ }^{5,7}$ and pathologic findings, ${ }^{26}$ we performed further shape analysis of the bilateral GP, putamen, and caudate nucleus. FDR-cor- rected statistical tests revealed pronounced focal differences in the shape of the GP and putamen in the Child B and Child C groups, compared with the controls. The shape of the left caudate nucleus also differed in patients with Child C compared with controls.

Putamen Shape. The most prominent shape alterations observed in the putamen were an inward deformation in the moderate-sized regions of the anterior and posterior aspects of the lateral putamen bilaterally and an inward deformation along the center of the medial putamen. Shape abnormalities were more prominent in patients with Child C (Fig 2).

Caudate Shape. The most prominent shape alterations were found in the left caudate of patients with Child $\mathrm{C}$ and involved an inward deformation in the lateral caudate head (Fig 3). 


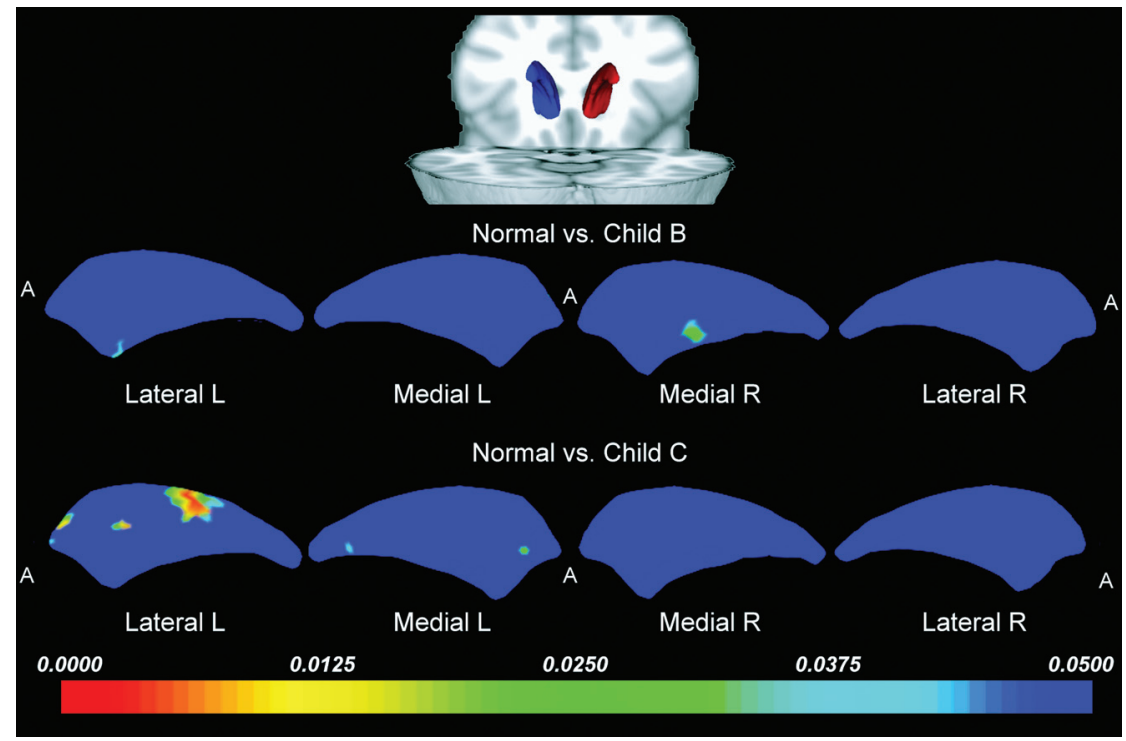

Fig 3. Statistical $3 D$ maps revealing significant caudate nucleus volume reduction in the left lateral aspect in the Child $C$ group versus controls. $R$ indicates right; $L$, left; $A$, anterior.

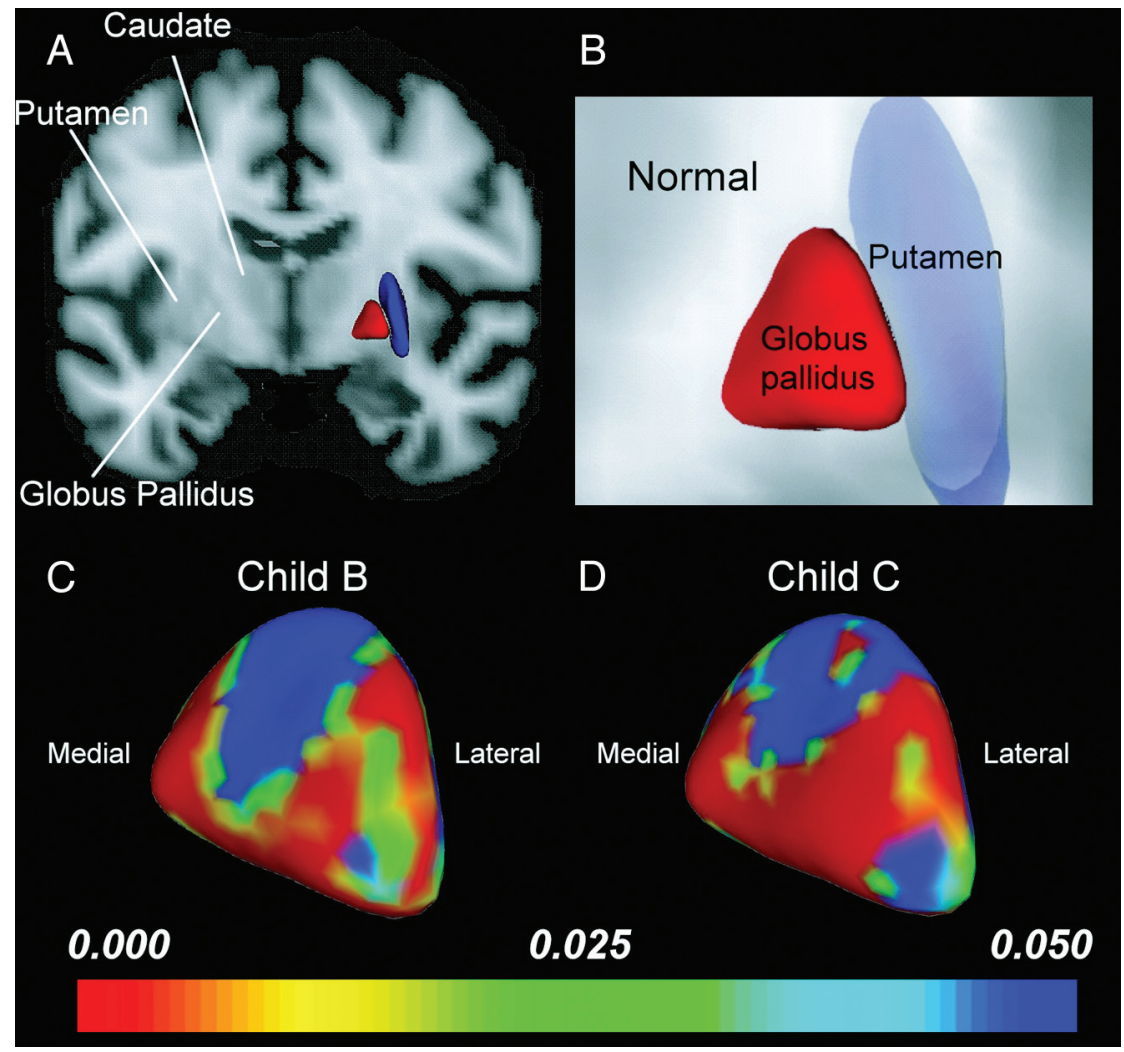

Fig 4. Shape analysis of the GP. $A$ and $B$, Anterior coronal views display the relative anatomic location of the putamen and GP in a healthy control. $C$ and $D$, Statistical $3 D$ maps display right pallidal volume changes with $P$ values color-coded at each surface point in the Child $B(C)$ and Child $C$ groups $(D)$. Note that the GP volume decrease in patients with cirrhosis, while not directly measured, is indicated by the location of the shape change. Medial expansion and lateral compression of the GP was found to be proportional to the severity of liver cirrhosis and further suggested counterbalancing roles between the medial and lateral GP physiologically.

GP Shape. A smaller GP in patients with cirrhosis, while not directly measured, is indicated by the location of the shape change. Patients with both Child B and Child C showed focalshape differences in the bilateral GP compared with control patients. The most prominent shape-change patterns in the GP were an expansion deformation along the medial GP facing the internal capsule and a compression deformation of the lateral GP facing the putamen (Fig 4).

\section{Association of Demographic and Clinical Variables on} DGM Volume

The left putaminal volume change $(F=12.96, P<.001)$ was best predicted by the pallidal index, $(\beta=-0.560)$, followed by TIV $(\beta=0.412)$ and the INR value $(\beta=$ $-279)$. The right putaminal volume change $(F=17.71, P<$ $.001)$ was best predicted by the pallidal index $(\beta=-0.612)$, followed by TIV $(\beta=0.520)$. The left caudate nucleus vol- 


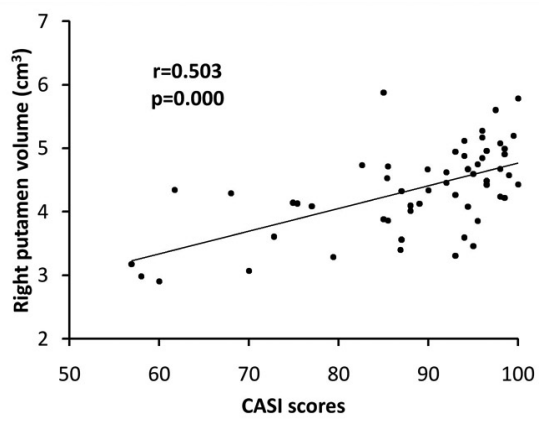

Fig 5. The relationship between total CASI scores and volume of the bilateral putamina

ume change $(F=8.082, P=.002)$ was best predicted by TIV $(\beta=0.534)$, followed by the pallidal index $(\beta=$ $-0.381)$.

\section{Decreased DGM Volume Correlated to Poorer Cognitive Test Results}

Lower CASI scores were positively associated with lower volume in the right putamen $(r=0.503, P<.001)$, left putamen $(r=0.466, P<.001)$ (Fig 5), and left nucleus accumbens $(r=$ $0.294, P=.034)$. Further analyses revealed that lower DGM volumes were also associated with subtests of CASI and are shown in the On-line Table.

\section{Discussion}

We identified significant local shape alterations in the GP, putamen, and caudate nucleus in patients with chronic liver disease as well as the expected findings of lower volumes of the putamen, caudate nucleus, and amygdala. A smaller volume was proportional to the severity of the liver disease. The higher pallidal index and higher INR values also correlated with a lower putaminal and caudate nucleus volume, which were linearly related to worse cognitive performance. To our knowledge, this is the first study explicitly correlating a lower volume of the putamen and caudate nucleus with the severity of chronic liver disease.

The toxicity of Mn to the BG has also been demonstrated in primates after repeated $\mathrm{Mn}$ administration ${ }^{27}$ and in workers exposed to $\mathrm{Mn}^{28}$ with identical imaging results. Oxidative stress and mitochondria play major roles in the Mn-induced degenerative process. ${ }^{29}$ The Mn dose and oxidation state also affect a number of brain outcomes, including the levels of neurotransmitter and proteins involved in cellular iron metabolism. ${ }^{30}$ Histopathologic evaluation of the brain tissues of patients with cirrhosis revealed astrocytosis, a neuropathologic finding particularly prevalent in the BG. ${ }^{31}$ This prominent gliosis and neuronal loss alter the BG structure. ${ }^{32}$ Currently, the pallidal index, indicative of pathologic $\mathrm{Mn}$ accumulation, accurately predicted a smaller volume in 3 DGM structure, suggesting a strong causal relationship.

Other factors in patients with liver disease might also contribute to the lower DGM volume. ${ }^{11}$ Malnutrition was associated with brain atrophy in cases of both alcoholic and viral cirrhosis. ${ }^{33}$ In this study, the relationship between the INR value and the subcortical volume may represent long-term malnutrition and vitamin $\mathrm{K}$ and other oligoelement deficits in end-stage liver disease. ${ }^{34}$

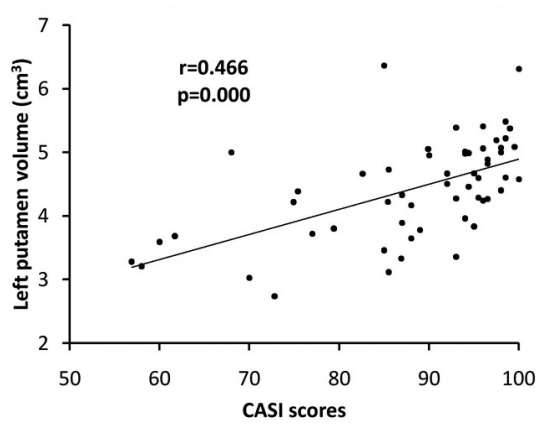

The significant lower putaminal volume in patients with Child B suggests that degenerative pathology affects this structure more or earlier in liver cirrhosis than the other DGM areas. Neuronal damage increased significantly, and more regions of the DGM were affected as the disease progressed, indicating a strong positive correlation. The results also suggest that some structures are relatively preserved in chronic liver disease (thalamus, nucleus accumbens, and hippocampus).

Neuroplasticity allows local structural shape changes without the presence of significant volumetric differences. ${ }^{12,15,16}$ Surprisingly, there was no significant GP volume reduction in patients compared with controls. This contradicts the known radiologic and pathologic findings. Nevertheless, significant surface shape alteration was found in the medial and lateral GP (Fig 4). The underlying mechanism of GP regional shape alteration is unclear at the present time. The neurotoxic effect of Mn deposition is most prominent in the GP with a significant loss of nerve cells, ${ }^{35}$ which were marked in the medial segments and moderate in the lateral ones. It also displayed a moderate increase of enlarged Alzheimer type II astrocytes with mild proliferation of glial fibers. ${ }^{31,35}$ In the putamen and caudate nucleus, the large nerve cells showed a moderate decrease in number, with shrinkage of those remaining. Significant volume reduction in the ventral putamen and lateral GP was consistent with the histopathologic findings. However, whether medial GP expansion correlated with increased larger astrocytes and glial fiber proliferation may not be fully addressed by our study.

Functionally, the medial and lateral GP play counterbalancing roles in complex motor programs. ${ }^{36}$ As a result, reduced nigrostriatal dopamine levels (lesions of the external GP and striatum) lead to disinhibition of the subthalamic nucleus and medial GP. ${ }^{4}$ Astrocytes in the BG may be regionally differentiated functionally, and the morphologic evolution of the GP supports such neural feedback circuitry. The reduced inhibition input from the damaged striatum and external GP (a compression deformity) with subsequent expansion of the internal GP provides the possible explanation.

This study has several limitations. Significantly ill patients were excluded from MR imaging for safety; therefore, whether additional volume reduction of the subcortical nuclei occurs is unknown. A higher pallidal index was associated with smaller DGM. However, the correlation between DGM degradation and serum $\mathrm{Mn}$ concentration is unclear. If a significant corre- 
lation exists, efforts to reduce serum Mn levels should be encouraged. Without complete neuropsychiatric and movement-disorder examinations, our data were interpreted indirectly from other studies. Studies examining specific associations between various degrees of focal brain structure alteration and clinical symptoms should be conducted in the future. Although the accuracy of each participant's native space subcortical segments was verified by a trained radiologist, the elevated MR imaging signal intensity in the BG possibly influences the reliability of the segmentation. Further validation is required.

\section{Conclusions}

We demonstrated volume reduction that developed in the BG according to the severity of liver cirrhosis. Furthermore, the pallidal hyperintensity independently predicted the degree of BG volume loss, which was linearly related to worse cognitive performance. Finally, we revealed that nonuniformly distributed shape abnormalities in the striatum stressed the value of additional psychomotor research in patients with cirrhosis.

\section{Acknowledgments}

The authors acknowledge MR imaging support from the MR imaging Core Facility, Chang Gung Memorial Hospital. We also thank Tsui-Min Chiu, Ping-Yang Yeh, Yu-Hsin Hsieh, and all subjects who participated in this study.

\section{References}

1. Joebges EM, Heidemann M, Schimke N, et al. Bradykinesia in minimal hepatic encephalopathy is due to disturbances in movement initiation. $J$ Hepatol 2003;38:273-80

2. Spahr L, Vingerhoets F, Lazeyras F, et al. Magnetic resonance imaging and proton spectroscopic alterations correlate with parkinsonian signs in patients with cirrhosis. Gastroenterology 2000;119:774-81

3. Jover R, Company L, Gutierrez A, et al. Clinical significance of extrapyramidal signs in patients with cirrhosis. J Hepatol 2005;42:659-65

4. Bhatia KP, Marsden CD. The behavioural and motor consequences of focal lesions of the basal ganglia in man. Brain 1994;117(Pt 4):859-76

5. Kulisevsky J, Pujol J, Balanzo J, et al. Pallidal hyperintensity on magnetic resonance imaging in cirrhotic patients: clinical correlations. Hepatology 1992;16:1382-88

6. Rovira A, Cordoba J, Sanpedro F, et al. Normalization of T2 signal abnormalities in hemispheric white matter with liver transplant. Neurology 2002;59:335-41

7. Pujol A, Graus F, Peri J, et al. Hyperintensity in the globus pallidus on T1weighted and inversion-recovery MRI: a possible marker of advanced liver disease. Neurology 1991;41:1526-27

8. Klos KJ, Ahlskog JE, Kumar N, et al. Brain metal concentrations in chronic liver failure patients with pallidal T1 MRI hyperintensity. Neurology 2006;67:1984-89

9. Lodi R, Tonon C, Stracciari A, et al. Diffusion MRI shows increased water apparent diffusion coefficient in the brains of cirrhotics. Neurology 2004;62:762-66

10. Saksena S, Rai V, Saraswat VA, et al. Cerebral diffusion tensor imaging and in vivo proton magnetic resonance spectroscopy in patients with fulminant hepatic failure. J Gastroenterol Hepatol 2008;23:e111-19

11. Jones EA, Weissenborn K. Neurology and the liver. J Neurol Neurosurg Psychiatry 1997;63:279-93
12. Qiu A, Crocetti D, Adler M, et al. Basal ganglia volume and shape in children with attention deficit hyperactivity disorder. Am J Psychiatry 2009;166:74-82

13. Gur RC, Gunning-Dixon FM, Turetsky BI, et al. Brain region and sex differences in age association with brain volume: a quantitative MRI study of healthy young adults. Am J Geriatr Psychiatry 2002;10:72-80

14. de Jong LW, van der Hiele K, Veer IM, et al. Strongly reduced volumes of putamen and thalamus in Alzheimer's disease: an MRI study. Brain 2008; 131:3277-85

15. Hwang J, Lyoo IK, Dager SR, et al. Basal ganglia shape alterations in bipolar disorder. Am J Psychiatry 2006;163:276-85

16. Levitt JJ, Styner M, Niethammer M, et al. Shape abnormalities of caudate nucleus in schizotypal personality disorder. Schizophr Res 2009;110:127-39

17. Mechtcheriakov S, Brenneis C, Egger K, et al. A widespread distinct pattern of cerebral atrophy in patients with alcohol addiction revealed by voxel-based morphometry. J Neurol Neurosurg Psychiatry 2007;78:610-14

18. Atterbury CE, Maddrey WC, Conn HO. Neomycin-sorbitol and lactulose in the treatment of acute portal-systemic encephalopathy. a controlled, doubleblind clinical trial. Am J Dig Dis 1978;23:398-406

19. Kale RA, Gupta RK, Saraswat VA, et al. Demonstration of interstitial cerebral edema with diffusion tensor MR imaging in type $\mathrm{C}$ hepatic encephalopathy. Hepatology 2006;43:698-706

20. Pugh RN, Murray-Lyon IM, Dawson JL, et al. Transection of the oesophagus for bleeding oesophageal varices. Br J Surg 1973;60:646-49

21. Lin KN, Wang PN, Liu CY, et al. Cutoff scores of the cognitive abilities screening instrument, Chinese version in screening of dementia. Dement Geriatr Cogn Disord 2002;14:176-82

22. Smith SM, Jenkinson M, Woolrich MW, et al. Advances in functional and structural MR image analysis and implementation as FSL. Neuroimage 2004; 23(suppl 1):S208-19

23. Patenaude B, Smith S, Kennedy D, et al. Bayesian shape and appearance models: FMRIB Technical Report TR07BP1. http://www.fmrib.ox.ac.uk/analysis/techrep. Accessed 2007

24. Csernansky JG, Joshi S, Wang L, et al. Hippocampal morphometry in schizophrenia by high dimensional brain mapping. Proc Natl Acad Sci U A 1998;95:11406-11

25. Park NH, Park JK, Choi Y, et al. Whole blood manganese correlates with high signal intensities on T1-weighted MRI in patients with liver cirrhosis. Neurotoxicology 2003;24:909-15

26. Rose C, Butterworth RF, Zayed J, et al. Manganese deposition in basal ganglia structures results from both portal-systemic shunting and liver dysfunction. Gastroenterology 1999;117:640-44

27. Pentschew A, Ebner FF, Kovatch RM. Experimental manganese encephalopathy in monkeys: a preliminary report. J Neuropathol Exp Neurol 1963;22:488-99

28. Kim Y, Kim KS, Yang JS, et al. Increase in signal intensities on T1-weighted magnetic resonance images in asymptomatic manganese-exposed workers. Neurotoxicology 1999;20:901-07

29. Dobson AW, Erikson KM, Aschner M. Manganese neurotoxicity. Ann N Y Acad Sci 2004;1012:115-28

30. Reaney SH, Bench G, Smith DR. Brain accumulation and toxicity of Mn(II) and Mn(III) exposures. Toxicol Sci 2006;93:114-24

31. Butterworth RF, Giguere JF, Michaud J, et al. Ammonia: key factor in the pathogenesis of hepatic encephalopathy. Neurochem Pathol 1987;6:1-12

32. Maeda H, Sato M, Yoshikawa A, et al. Brain MR imaging in patients with hepatic cirrhosis: relationship between high intensity signal in basal ganglia on T1-weighted images and elemental concentrations in brain. Neuroradiology 1997;39:546-50

33. Plauth M, Merli M, Kondrup J, et al. ESPEN guidelines for nutrition in liver disease and transplantation. Clin Nutr 1997;16:43-55

34. Harrington DJ, Western H, Seton-Jones C, et al. A study of the prevalence of vitamin $\mathrm{K}$ deficiency in patients with cancer referred to a hospital palliative care team and its association with abnormal haemostasis. J Clin Pathol 2008; 61:537-40. Epub 2007 Oct 8

35. Yamada M, Ohno S, Okayasu I, et al. Chronic manganese poisoning: a neuropathological study with determination of manganese distribution in the brain. Acta Neuropathol 1986;70:273-78

36. Crossman AR, Peggs D, Boyce S, et al. Effect of the NMDA antagonist MK-801 on MPTP-induced parkinsonism in the monkey. Neuropharmacology 1989;28:1271-73 